POS $\quad$ PROCEEDINGS

\title{
Study on the Remote Control of WFCTA for LHAASO
}

\author{
Fengrong Zhu *i \\ School of Physical Science and Technology, Southwest Jiaotong University, Chengdu 610031, \\ P.R. China, E-mail: zhufr@home.swjtu.edu.cn
}

\section{Yong Zhang}

Key Laboratory of Particle Astrophysics, Institute of High Energy Physics, Chinese Academy of Science, P.O. Box 918, 100049 Beijing, China

\section{Ning Xie}

School of Physical Science and Technology, Southwest Jiaotong University, Chengdu 610031, China

\section{Huanyu Jia}

School of Physical Science and Technology, Southwest Jiaotong University, Chengdu 610031, China

\section{Xiumei Li,}

School of Physical Science and Technology, Southwest Jiaotong University, Chengdu 610031, China

\begin{abstract}
Wide Field CherenKov Telescope Array(WFCTA) of LHAASO, consisting of 12 telescopes, has been proposed to be mounted at Daocheng County, Sichuan Province, China, to probe the cosmic rays with the energy of $3 \cdot 10^{13} \mathrm{eV}-10^{17} \mathrm{eV}$ together with WCDA and KM2A of LHAASO. 2 YAG lasers, $2 \mathrm{~N} 2$ lasers and 2 WFCTA prototypes will be built to calibrate the WFCTA. 1 YAG laser will be placed at a site far away from the LHAASO about $10 \mathrm{~km}$ to measure horizontal attenuation length of aerosol, while cable internet can not be used. To control remotely the computer of the laser lidar, the various softwares, like Sunlogin, TeamViewer have been tried to install in Windows, Scientific Linux and Ubuntu Linux operating system, the test results about the stability, upload and download speed to determine the remotely controlling approch for the WFCTA of LHAASO will be reported in this presentation.
\end{abstract}

35th International Cosmic Ray Conference - ICRC2017

10-20 July, 2017

Bexco, Busan, Korea

\footnotetext{
* Speaker.

${ }^{\dagger}$ on behalf of the LHAASO Collaboration.
} 


\section{Introduction}

The Large High Altitude Air Shower Observatory (LHAASO) is a multi-objective project to probe the origin of cosmic rays with a complicated $1.2 \mathrm{~km}^{2}$ ground-based detector array [1]. The total three components are KM2A[2, 3], WCDA [4] and WFCTA[5]. And KM2A is one kilometer square extensive air shower array, designed for the measurement of the number density and arrival time of shower particles, contains 5242 EDs (Electromagnetic particle Detectors) and 1221 MDs (Muon Detectors). WCDA, the water Cherenkov detector array, with an area of $78000 \mathrm{~m}^{2}$ and 350000 tons of purified water, is planned to be built in a couple of years at Mount Haizi, Daocheng, at an altitude of $4410 \mathrm{~m}$ a.s.l. The main purpose of the WCDA is to survey the northern sky for VHE gamma ray sources. WFCTA, consiting of 12 telescopes, has been proposed to mounted at Daocheng County, Sichuan Province, China. Its major goal is to probe the galactic cosmic rays with the ultra energy of $3 \times 10^{13} \mathrm{eV}-10^{17} \mathrm{eV}$ together with WCDA and KM2A of LHAASO.

The calibration system for the WFCTA is to be built with a series of devices, mainly including two WFCTA prototypes, 2 YAG lasers, 2 Nitrogen lasers with high precisly revolving table, an infrared thermometer and a meteorological station. With this calibration system we can study absolute calibration of photon number, air quality monitoring and back scatter method monitoring weather, also study the measurement of the horizontal attenuation length, distribution of aerosol scatter angle, aerosol effective scale height. In order to precisely measure of the horizontal attenuation length, 1 YAG laser system need to be mounted a site of about 10 kilometeres away from the LHAASO, where the cable internet probably not be available in the future according to the overall plan of the construction of LHAASO, so the remote computer of the YAG laser lidar can not be controled by TCP-IP protocol. In oder to transfer calibration data to be taken by laser lidar to Chengdu, China or Beijing, China by internet communication. It is necessary for us how to control of remote computer by softwares installed in Windows, Scientific Linux system or other operating systems. In this presentation we will simply introduce the remote control softwares of Sunlogin and TeamViewer in Section 2, and in Section 3 the internet speed test results in Chengdu City are presented, Section 4 is the test results of internet speed and transmission speed in Daocheng, China, and the last is the summary.

\section{Remote-control software and the tool for the test of network speed}

Sunlogin and TeamViewer software are commonly remote-control softwares in China. TeamViewer is better than Sunlogin in compatibility and Sucerity. Sunlogin is more favourable in Window operating system with client, multi-function, and remote desktop, remote video recording file, but in Linux operating system it is limited, like only being controled by other computer, not control other computer, especially it can not transmit file from a computer to another computer in any linux operating system.

Our study shows Sunlogin can be installed in Ubuntu16.04, but it is dependent on the architecture of the operating system and our system need to be fixed 32-bit memory as shown in Figure1. Also Sunlogin can not be installed in Scientific Linux Cern system.

TeamViewer has many features, fisrtly of all, it can run in Cross-platform PC to PC, mobile to PC, PC to mobile, and even mobile to mobile connections that support Windows, macOS, Linux, 


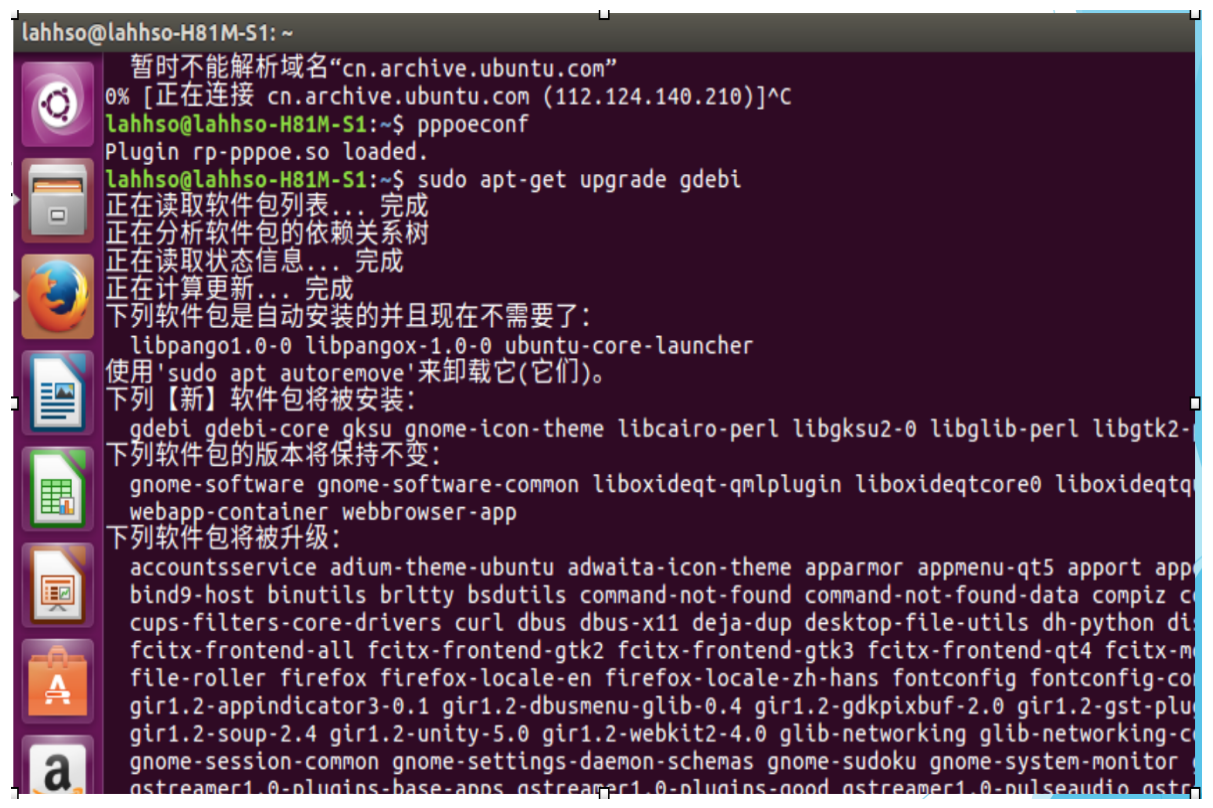

Figure 1: architecture modified to run Sunlogin

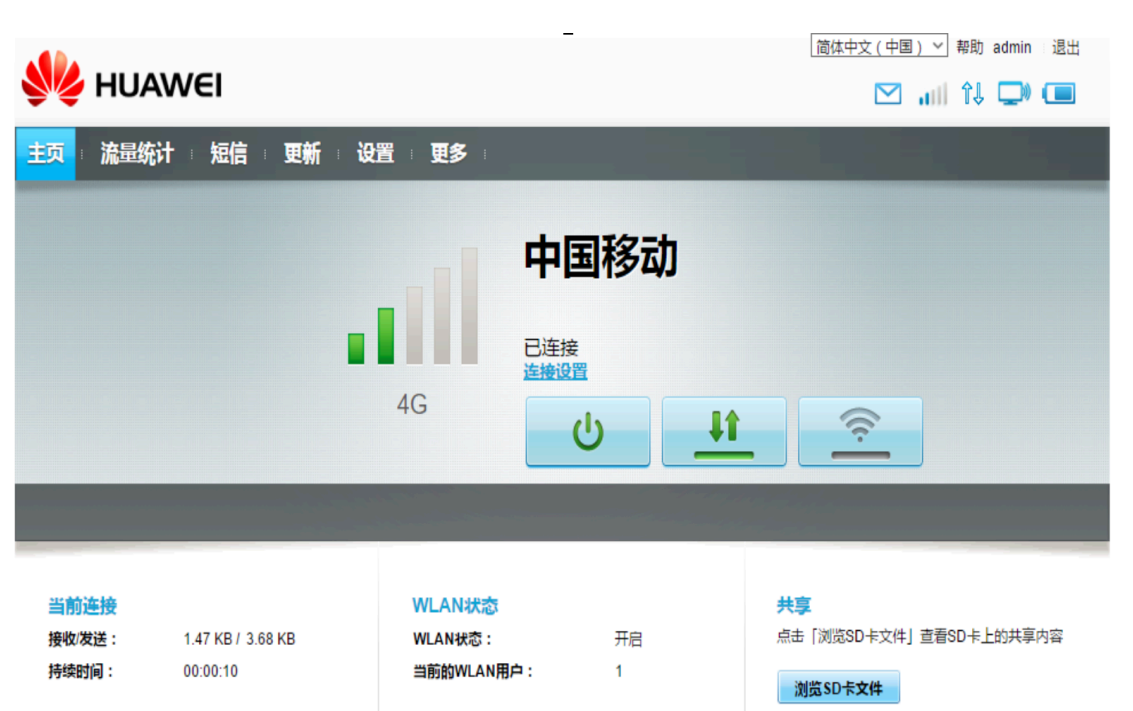

Figure 2: Normal status of Huawei E5573 Mobile Wi-Fi

Chrome OS, iOS, Android, Windows Universal Platform, and Black Berry. Secondly we can start TeamViewer instantly. TeamViewer even works behind firewalls and automatically detects any proxy configuration. Thirdly it has high performance, such as intelligent connection setup and routing, efficient use of bandwidth, fast data transmissions, remote session framerates up to $60 \mathrm{fps}$, and automatic quality adjustments ensure an optimized user experience. It has other advantages like no configuration, easy to understand and high security. It needs to know that TeamViewer software is also dependent on the architecture of the system. More detailed information on the 


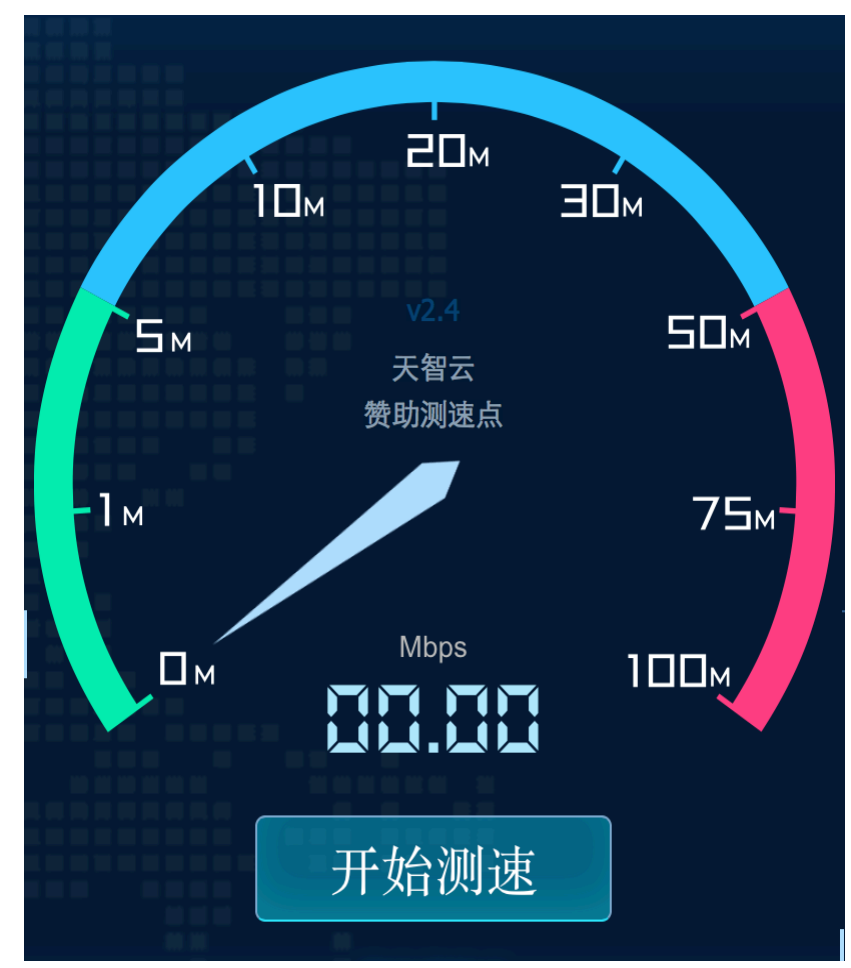

Figure 3: Visual network speed test tool

installation and usage can be found in the paper of [6]

For the comparation based on our experience, TeamViewer is better than Sunlogin in compatibility and Sucerity. the Sunlogin can run in Windows and Ubuntu 16.04 linux operating system, but not in Scientific Linux operating system, also it is not stable and can not transfer files in Linux operating system. TeamViewer software can run stably in Ubuntu16.04, Scientific Linux 7.2 and also can transimit files in these systems.

We select the web page of Speedtest.net to test the speed of network and transmissing of files. The Speedtest.net is a famous global broadband speed test website and distributed in hundred of nodes around the world. It can be used with only browser, not to download and install any softwares.This Speedtest tool is online and visual as shwon in Figure 3. The number of download and upload speed will show in the disk when pressing the button of "start test".

\section{Network speed test in Chengdu City}

For the wireless internet signal it is stronger in central city than in remote area like in Daocheng. Jiuli Campus of Southwest Jiaotong University located in central north of Chengdu City, is easy to receive the communication network signal with SIM telephone card by China Mobile and China Unicom . For the router we selected Huawei E5573 4G Mobile Wi-Fi with download speeds of up to $150 \mathrm{Mbps}$ and upload speeds of up to $50 \mathrm{Mbps}$ shown in Figure 2. 

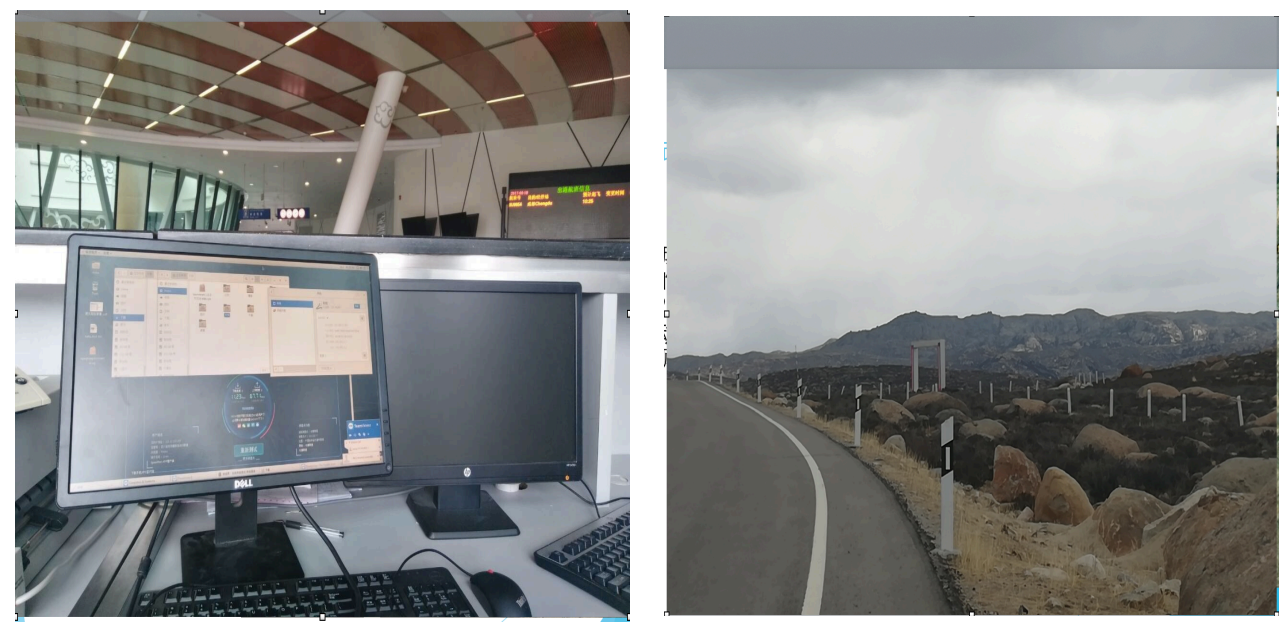

Figure 4: left: Aden Daocheng Airport test site, right: LHAASO base test site

The performance of Sunlogin software in Ubuntu 16.04 linux operating system were tested in the cable network and wireless internet. It is interesting that the internet speed in Mobile Wi-Fi mode is faster than in cable internet mode. The mean values of download and upload speed were 1.071Mbps and 0.122 Mbps in Mobile Wi-Fi mode while the mean values of download and upload speed were 0.404Mpbs and 0.7Mbps according to our records in March 2017.

\section{Network speed and files transmission test in Daocheng County}

\subsection{Test method}

In order to succesfully tranfer the calibration data of WFCTA to our university, it is the key to have a smooth speed of internet at Daocheng. For the Wireless network test, we adapted the SIM card from China Unicom and China Mobile employed at Chengdu, and the same Huawei E5573 4G Mobile Wi-Fi.

TeamViewer software was tested at Aden Daocheng Airport hall(site 1), shown in Figure 4(left), which is about $10 \mathrm{~km}$ far away of LHAASO base and mounted cable network to get internet. We use a PC installed Ubuntu 16.04 and Scientific Linux 7.2 system. At the same time inside LHAASO base, the Huawei E5573 Mobile Wi-Fi with mobile card received no any signal from China Mobile and China Unicom, but at a nearby roadside(site 2, shown in Figure 4(right)), about $500 \mathrm{~m}$ away of the LHAASO base showed there was internet singal from only China Unicom.

At this site we tested Sunlogin software with a laptop installed only Windows. For the two remote controls mode, the host control was done in Chengdu City, China $800 \mathrm{~km}$ away from the LHAASO base. 


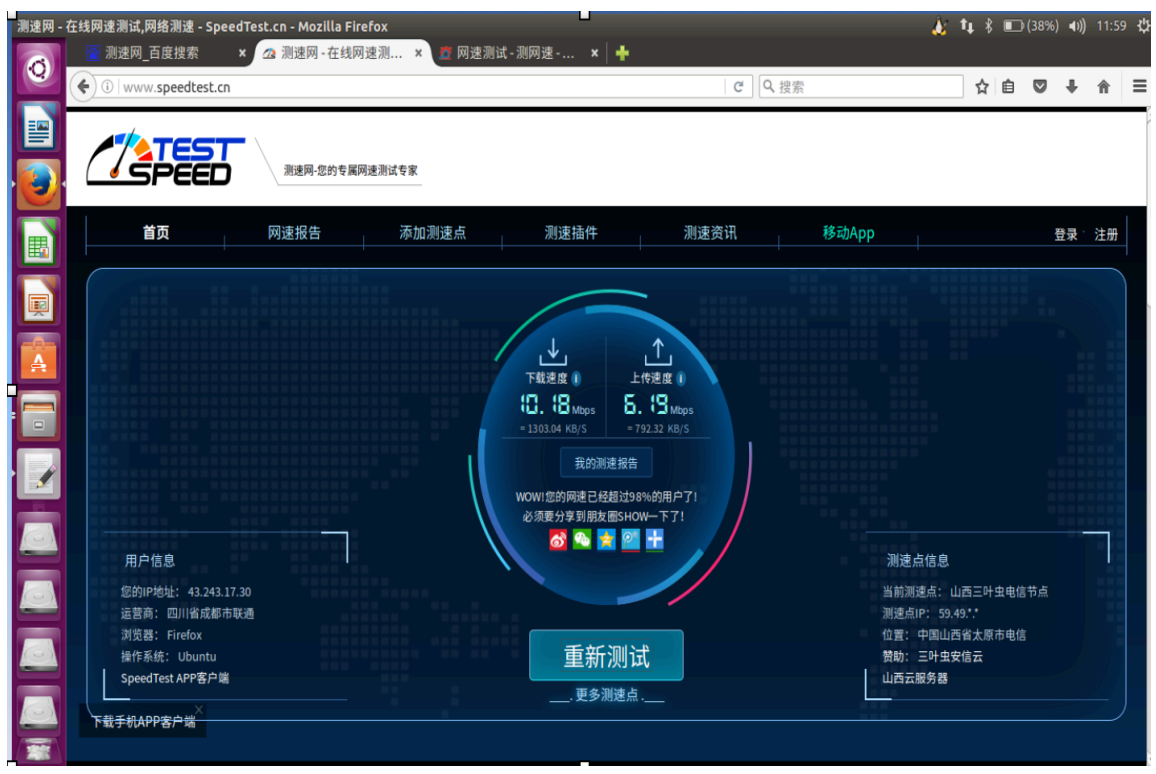

Figure 5: Speed test web page, the IP address:43.243.17.30, the operator is Chengdu UnicomiijNNSichuan Province. The brower is Firefox and operating system is Ubuntu. The download speed $10.18 \mathrm{Mbps}$ and upload speed 6.19 Mbps shown inside of the circle in the middle of the web page.

\subsection{Test results}

The speed of network is very important for the transmissions of lots of WFCTA calibration data, we tested the internet speed at the two sites after internet connection shown in Figure 5 with http://www.speedtest.cn.

There are some information on the client of the user, the IP address:43.243.17.30, the operator is Chengdu Unicom, Sichuan Province. The brower is Firefox and the operating system is Ubuntu. The download speed 10.18 Mbps and upload speed 6.19 Mbps shown inside of the circle in the middle of the web page. At site 1 the average of the download speed in 2 hours is $554 \mathrm{~Kb} / \mathrm{s}$ and the upload speed is $915 \mathrm{~Kb} / \mathrm{s}$, seperately. At site 2 the average of the download speed in 2 hours and the upload speed is $7332 \mathrm{~Kb} / \mathrm{s}$ and $1086 \mathrm{~Kb} / \mathrm{s}$. Also the network was smooth and stable during our test at the two sites.

The speed of files transmission at the two sites were also tested with the TeamViewer, a file is uploading to the computer of jjyThinePad-E550c as shown in Figure 6, and the upload speed is $170 \mathrm{~kb} / \mathrm{s}$. In right-down corcer the host computer information is listed here. At site 1 the download speed of file transmission in Scientific operating system is $240 \mathrm{~Kb} / \mathrm{s}$, while the upload speed is 120 $\mathrm{Kb} / \mathrm{s}$. At site 2 the download speed of file transmission in Ubuntu 16.04 operating system is 110 $\mathrm{Kb} / \mathrm{s}$, while the upload speed is $110 \mathrm{~Kb} / \mathrm{s}$. 


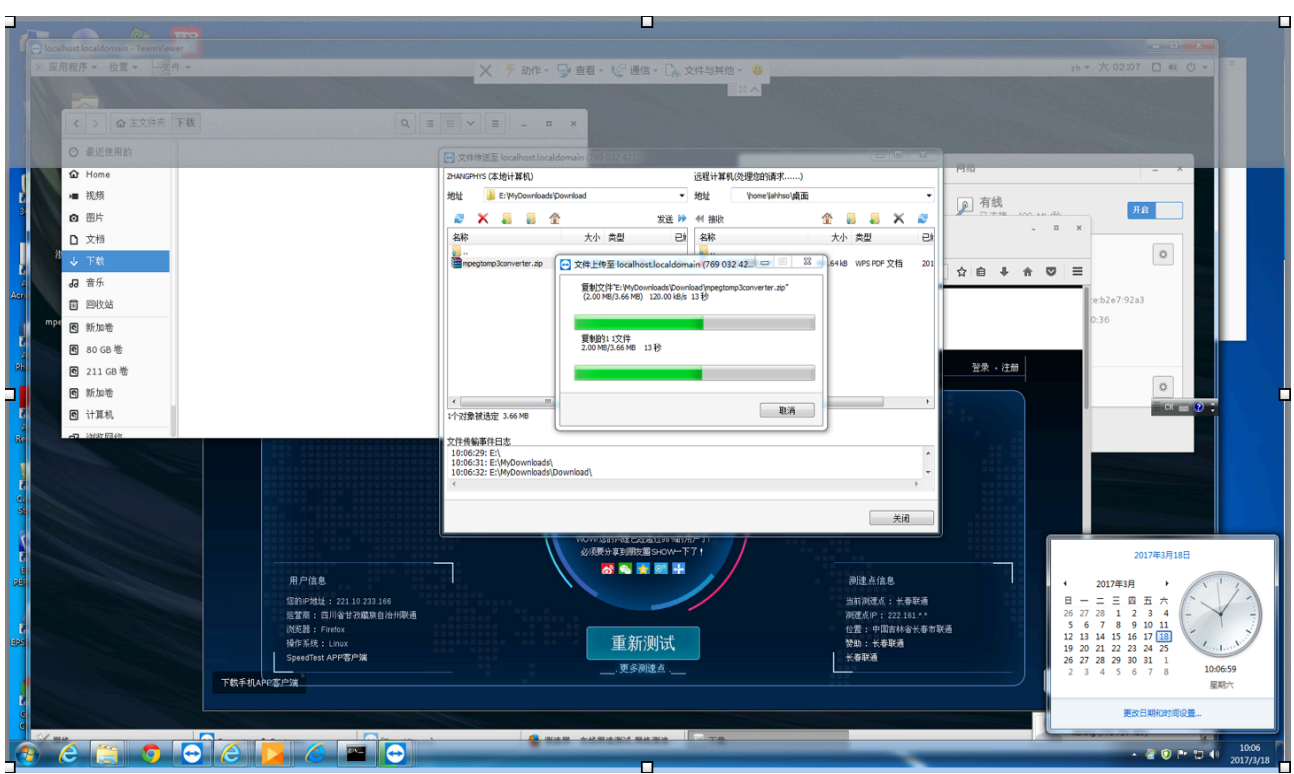

Figure 6: an example of files transmission under the remote control of TeamViewer in Scientific Linux operating system from Daocheng County to Chengdu City during our test.

\section{Summary and Conclusion}

We suceeded to test the network speed at the campus of Southwest Jiaotong University in wireless and wire communication, seperately, and the wireless communication is faster than the expected. Also the remote-control TeamViewer software can reach our demand in good network communication, but the Sunlogin software sometimes can not be logged during our test, so it is not so stable, which suggests to adapt the TeamViewer software in Scientific Linux operating system for the WFCTA of LHAASO in the future. It is lucky that the communication signal nearby the LHAASO base from China Union is enough to tranfer files fastly and safely and we can use it in the future.

\section{Knowledgement}

This work is supported by National Natural Science Foundation (NSFC) of China under contacts No. 11635011 and 11205126

\section{References}

[1] Cao, Z. (for the LHAASO Collaboration) 2010, Chin. Phys. C, 34, 249

[2] Xu Wang, Zhong-Quan Zhang, Ye Tian et al. 2016, Chin. Phys. C, 40(8),86003

[3] ZHAO Jing;LIU Jia;SHENG Xiang-Dong et al. 2014, Chin. Phys. C, 38(11),36002

[4] Cong Ma, Lei Zhao, Yu-Xiang Guo, Jian-Feng Liu, Shu-Bin Liu, Qi An. 2016, Chin. Phys. C,40 (1) 16101

[5] MA Ling-Ling;BAI Yun-Xiang;CAO Zhen et al. 2011, Chin. Phys. C,35(5) 478 
[6] Wang Pinggang, Fan Peipei, 2014, Electronic Technology and Software Engineering,13,131 